\title{
COMPORTEMENT DE DIVERSES PROVENANCES DE MÉLEZZES EN BRETAGNE
}

\author{
PAR \\ J. - F. LACAZE \\ Ingénieur des Eaux et Forêts \\ et \\ M. LEMOINE \\ Ingénieur Agronome \\ (Station d'Amélioration des arbres forestiers - CNRF)
}



L'existence de races différentes à l'intérieur de l'espèce Larix decidua Miller a alimenté une littérature abondante. Divers auteurs ont décrit des caractères morphologiques susceptibles de distinguer certaines de ces races (d'où la création de sous-espèces); mais surtout, de nombreux documents mettent en évidence des différences marquées dans leur comportement lorsqu'elles sont introduites hors de leur aire naturelle.

Le présent article a pour but de donner les premiers résultats fournis par deux plantations comparatives de provenances de mélèzes d'Europe, installées en Bretagne (Forêt domaniale de Coat-anHay) à l'automne 1959 dans le cadre d'une expérience internationale organisée par le Professeur Scrober (Hann-Münden).

\section{1 - Description des principales provenances expérimentées.}

L'aire du mélèze d'Europe a été décrite en détail par FourCHY (1), aussi nous nous contenterons de rappeler brièvement qu'il s'agit d'une aire fractionnée en quatre zones géographiques principales d'inégale importance: Alpes, Sudètes, Tatras, Pologne. (Voir carte.)

On trouvera sur le tableau I une description sommaire des provenances installées à Coat-an-Hay auxquelles s'ajoutent à titre de témoins, une provenance de mélèze du Japon (Larix leptolepis Murr.) et 1'hybride (Larix $\times$ eurolepis A. Henry) de deux sources différentes (anglaise et danoise). T,es hybrides danois ont été créés à l'initiative du Professeur Syrach Larsen. Les subdivisions de la zone géographique principale données sur le tableau I ont été établies par le Professeur ScHober.

L'échantillonnage de provenances de mélèze d'Europe comporte quelques lacunes graves:

Pour l'aire alpine, ces lacunes concernent: les Alpes centrales suisses, les Alpes du Nord occidentales, et surtout la zone NordEst où le mélèze se développe à basse altitude (Wienerwald).

La zone géographique des Tatras est entièrement absente: une plantation comparative plus récente, installée à proximité des deux premières, lui est consacrée, avec une provenance du Wienerwald.

La zone géographique de la Pologne n'est représentée que par une seule provenance.

Enfin. les provenances artificielles correspondent à des peuplements remarquables, sélectionnés en Allemagne, France et Tchécoslovaquie et d'origine incertaine.

(1) Annales de l'Ecole Nationale dẹs Eaux et Forêts, 1952. 
TABLEAU I

DESCRIPTION DES PROVENANCES EXPÉRIMENTEES

\begin{tabular}{|c|c|c|c|c|c|c|c|c|c|c|}
\hline \multirow{2}{*}{ Zone géographi que } & \multirow{2}{*}{\begin{tabular}{|l|}
$N^{\circ}$ dea \\
prowe \\
nences
\end{tabular}} & \multirow{2}{*}{$\begin{array}{c}\text { Nom dos } \\
\text { provenancss }\end{array}$} & \multirow{2}{*}{ Tratitude } & \multirow{2}{*}{ Longitude } & \multirow{2}{*}{$\underset{(\mathrm{m})}{\text { Al titude }}$} & \multirow{2}{*}{$\underset{(\mathrm{mm})}{\text { Pluviosité }}$} & \multicolumn{2}{|c|}{ Température movense } & \multirow{2}{*}{$\begin{array}{c}N^{\circ} \text { de la } \\
\text { plentation } \\
\text { comparative }\end{array}$} & \multirow[b]{2}{*}{ Observations } \\
\hline & & & & & & & Anmuelle & $\begin{array}{l}\text { Mai के } \\
\text { Sent. }\end{array}$ & & \\
\hline Alpes maritimes françaises & 38 & Valdeblore & $44^{\circ} 14^{\prime} \mathrm{N}$ & $7^{\circ} 11 \mathrm{I} \mathrm{E}$ & $1700 / 1800$ & 1200 & 605 & 100 & P $300-2$ & \\
\hline Alpes du Sud-Ouest - Queyrae & 22 & Fmbrun Ristolas & $44^{\circ} 47^{\prime} \mathrm{N}$ & $6057 \cdot \overline{\mathrm{E}}$ & 1600 & 1050 & 601 & 7203 & P $300-1$ & \\
\hline Alpes du Sud-Ouest - Queyras & 23 & Rambrun Aiguilies & $44^{\circ} 47^{\prime} \mathrm{N}$ & $6^{\circ} 54^{\prime} \mathrm{E}$ & 1.560 & 1050 & 601 & 1203 & P $300-1$ & \\
\hline Alpes du Sud-Ouest - Briançonnais & 24 & Briançon Montgenâvre & $44056 \cdot \mathrm{N}$ & $60432 \mathrm{E}$ & 1730 & 808 & - & - & P $300-1$ & \\
\hline Alpes du Sud-Ouest - Briançonnais & 26 & Briançon Villard & $44^{\circ} 52^{3} \mathrm{~N}$ & $6039, \mathrm{E}$ & 1400 & 620 & 602 & 1301 & P $300-1$ & \\
\hline Alpes du Sud-Ouest - Briançonnais & 102 & Puy-St-Vincent & $44^{\circ} 48^{\prime} \mathrm{N}$ & $6032+E$ & 1850 & & & & P $300-1$ & \\
\hline Préalpes du Sud-Est - Val di Fiamme & 16 & Cavalèse & $46^{\circ} 19^{\prime} \mathrm{N}$ & $11027 \cdot \mathrm{E}$ & 1200 & 860 & 807 & 1608 & P $300-1$ et 2 & \\
\hline Préalpes du Sud-Est - Val Sugana & 18 & Tenna & $46004^{\prime} \mathrm{N}$ & $11^{\circ} 19^{1} \mathrm{E}$ & 600 & 1000 & $12^{\circ}$ & & P 300-1 & \\
\hline Préalpes du Sud-Est - Val Sugana & 20 & Cavedine & $45^{\circ} 59^{\prime} \mathrm{N}$ & $11^{\circ} 04^{\prime} \mathrm{F}$ & 600 à 700 & 1190 & $11^{\circ}$ & $16^{\circ}$ & P $300-1$ & \\
\hline Alpes intérieures - Tyrol & 2 & Schoenwies & $47^{\circ} 12^{\prime} \mathrm{N}$ & $100401 \mathrm{~F}$ & 1100 & & & & P $300-2$ & (1) \\
\hline Alpes intérieures - Tyrol du Sud & 12 & Sterzing/Flains & $46^{\circ} 54^{\prime} \mathrm{N}$ & $110261 \mathrm{E}$ & 1000 & 790 & $7^{\circ}$ & 1406 & P $300-2$ & (1) \\
\hline Alpes intérioures - Tauern. & 3 & Mithldorf & $46^{\circ} 52^{\prime} \mathrm{N}$ & $13021 \cdot \mathrm{E}$ & 900 & 1100 & $7^{\circ}$ & $14^{\circ 2}$ & P $300-1$ & \\
\hline Alpes du Nord & 4 & Fernpass & $47^{\circ} 22^{\prime} \mathrm{N}$ & $10^{\circ} 54^{\prime} \mathrm{E}$ & 1100 à 1200 & 1264 & $4^{\circ} 7$ & 1008 & P $300-2$ & (1) \\
\hline Alpes du Nord & 7 & Langau 38/41 & $47^{\circ} 49^{\prime} \mathrm{N}$ & $15^{\circ} 10^{\prime} \mathrm{E}$ & 1100 & 1780 & $3 \circ 9$ & $10^{\circ 9}$ & P $300-1$ & (1) \\
\hline Préalpes du Sud-Est & 11 & Wechselgebiet & $47^{030} \cdot \mathrm{N}$ & $16^{\circ} œ \ell^{\prime} \mathrm{E}$ & 1000 & 1028 & 601 & $13^{\circ} 1$ & P $300-1$ & \\
\hline Sudetes & 39 & Zabreh-Dubicko & $49050 \cdot \mathrm{N}$ & $16058^{\prime} \mathrm{E}$ & 400 & 670 & $7^{\circ} 5$ & $15^{\circ} \mathrm{I}$ & P $300-1$ & \\
\hline$n$ & 40 & Ruda nad Moravou & $49059 \cdot \mathrm{N}$ & $16^{\circ} 54^{\prime} \mathrm{E}$ & 480 & 670 & $7 \circ 9$ & $15^{\circ} 7$ & P $300-1$ et 2 & \\
\hline " & 106 & Olomouc & & & & & & & P 300-1 & \\
\hline " & 107 & Hradec nad Opavou & & & 400 & & & & P $300-1$ & \\
\hline Pologne & 104 & Grojec & $51^{\circ} 50^{\prime} \mathrm{N}$ & $20^{\circ} 45 \mathrm{l} \mathrm{B}$ & 180 & & & & P $300-1$ & \\
\hline Schleswig (artificiel) & 34 & Neumunster & $54^{\circ} 15^{\prime} \mathrm{N}$ & $10^{\circ} 10^{\prime} \mathrm{B}$ & 50 & 720 & $8^{\circ}$ & $14^{\circ} 4$ & P $300-1$ & \\
\hline Hesse（artificiel） & 28 & Schlitz 65 & $50^{\circ} 43 \cdot \mathrm{N}$ & $9031 \cdot \mathrm{E}$ & 335 & 634 & $7^{\circ} 8$ & $14^{\circ 2}$ & P $300-1$ & \\
\hline Tchécoslovaquie (artificioi) & 30 & Dobris & $49^{\circ} 47^{\prime} \mathrm{N}$ & $14^{\circ} 11^{\prime} \mathrm{E}$ & 500 & 590 & & & P $300-1$ & \\
\hline Alsace (artificiel) & 103 & Dumenach & $47^{\circ} 10^{\prime} \mathrm{N}$ & $7020 \cdot \mathrm{E}$ & 650 & 900 & $10^{\circ}$ & $17^{\circ}$ & P $300-2$ & \\
\hline Japon & 36 & Ins & $35^{\circ} 52^{\prime} \mathrm{N}$ & $138^{\circ} 04^{\prime} \mathrm{F}$ & 1200 & & & & P $300-1$ et 2 & (2) \\
\hline Ecosse & 108 & Dunkeld & & & & & & & P 300-1 & (3) \\
\hline Denemark & 110 & Humlebaeak & & & & & & & P $300-1$ & (5) \\
\hline$"$ & 109 & Hoersholm & & & & & & & P $300-2$ & (4) \\
\hline
\end{tabular}

(1) Graines récoltées séparément sur 7 arbres pour la provenance 2, 8 arbres pour la 12,5 arbres pour la 4 et 2 arbres pour la 7 .

(2) Larix leptolepis.

(3) Larix eurolepis - probablement une F2.

(4) Larix eurolepis - croisement contrôlé entre un clone de L. europaea (V 44) et un clone de L. Leptolepis (V 634).

(5) Larix eurolepis - verger à graines $n^{\circ} 201$ entre un clone de L. europaea (V 44) et les descendants de deux L. leptolepis sélectionnés 


\section{2 - Description de l'expérimentation.}

\section{1 - Description sommaire de la station.}

Les deux plantations comparatives ont été installées en forêt domaniale de Coat-an-Hay (Côtes-du-Nord) (1), après coupe rase d'un taillis-sous-futaie médiocre.

Deux raisons principales ont milité en faveur du choix d'une région à climat très atlantique: d'une part le désir de se placer sous des conditions très différentes de celles des autres plantations comparatives installées en Allemagne, Italie, Pays-Bas, Suède, Turquie et U.S.A. D'autre part, l'intérêt que le mélèze soulève en Bretagne, où les gros bois de cette espèce trouvent un débouché intéressant.

La forêt de Coat-an-Hay (altitude $200 \mathrm{~m}$ ), se situe à quelques kilomètres de Belle-Isle-en-Terre $\left(48^{\circ} 31^{\prime}\right.$ de latitude Nord, et $3^{\circ} 25^{\prime}$ de longitude Ouest). Le climat est caractérisé par:

- une pluviométrie de $890 \mathrm{~mm}$ (Station de Callac: $130 \mathrm{~m}$ d'altitude), très bien répartie dans l'année. L'état hygrométrique reste constamment élevé.

- une température moyenne annuelle de l'ordre de $10^{\circ} \mathrm{C}$ avec une très faible amplitude annuelle, quoique légèrement supérieure à celle de la station de Saint-Brieuc (106 m d'altitude) à laquelle nous nous référons :

\begin{tabular}{|c|c|c|}
\hline & $\begin{array}{l}\text { Pluviométrie } \\
\text { (mm) } \\
-\end{array}$ & $\begin{array}{c}\text { Température } \\
\left({ }^{\circ} \mathrm{C}\right) \\
-\end{array}$ \\
\hline Janvier $\quad \ldots \ldots \ldots \ldots \ldots \ldots$ & 86 & 4,7 \\
\hline Février $\quad \ldots \ldots \ldots \ldots \ldots \ldots$ & 69 & 5,4 \\
\hline Mars $\ldots \ldots \ldots \ldots \ldots \ldots \ldots$ & 67 & 6,7 \\
\hline Avril $\ldots \ldots \ldots \ldots \ldots \ldots \ldots$ & 60 & 9,4 \\
\hline 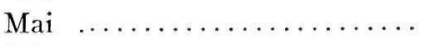 & 60 & 12,0 \\
\hline Juin $\ldots \ldots \ldots \ldots \ldots \ldots \ldots \ldots \ldots$ & 53 & 14,7 \\
\hline Juillet $\ldots \ldots \ldots \ldots \ldots \ldots \ldots$ & 60 & 16,5 \\
\hline Août $\ldots \ldots \ldots \ldots \ldots \ldots \ldots$ & 61 & 16,8 \\
\hline Septembre $\ldots \ldots \ldots \ldots \ldots \ldots$ & 75 & 14,9 \\
\hline Octobre $\ldots \ldots \ldots \ldots \ldots \ldots \ldots$ & 109 & 11,3 \\
\hline Novembre $\ldots \ldots \ldots \ldots \ldots$ & 98 & 7,8 \\
\hline Décembre $\ldots \ldots \ldots \ldots \ldots \ldots$ & 94 & 5,4 \\
\hline Total $\ldots$ & 892 & \\
\hline Moyenne ..... & & 10,5 \\
\hline
\end{tabular}

(1) Cette réalisation a été possible grâce à l'active collaboration de M. l'Ingénieur en Chef DE LA Fouchardiêre, des Chefs de District Ollivier et Jacouemin; le Chef de District Aubert, du C.N.R.F. y a également contribué. Qu'ils veuillent bien trouver ici l'expression de nos remerciements. 
Les gelées tardives sont fréquentes en particulier dans les basfonds (trous à gelée).

La roche mère (schiste) donne un sol brun assez léger favorable à la végétation forestière sauf en quelques zones mal drainées (parties basses également soumises à des gelées tardives).

\section{2 - Description de l'expérimentation.}

221 - Elevage des plants en pépinière (Amance, près de Nancy).

Le semis a été effectué le 23 avril 1958 selon un dispositif en blocs complets avec 2 répétitions.

Les semis ont été repiqués à 1 an, selon un dispositif en blocs complets à 3 répétitions, au printemps 1959, après un tri éliminant tous les plants dont la hauteur était inférieure à $70 \%$ de la movenne du lot.

\section{2 - Plantations comparatioes (Coat-an-Hay).}

La parcelle dévolue aux plantations fut l'objet d'une coupe rase suivie de l'extraction des souches au bulldozer. Cette dernière opération s'est révélée défavorable, une partie non négligeable de l'humus ayant été entrainée avec les souches.

L'installation des plants (1-1) a eu lieu en novembre 1959, selon l'espacement $2 \times 2 \mathrm{~m}$ dans un sol détrempé et au cours d'une période particulièrement pluvieuse.

\section{3 - Dispositifs expérimentanx.}

2231 - Expérience principale ( $\mathrm{P}, 300-1)$.

Les caractéristiques sont les suivantes:

- Blocs incomplets équilibrés

- 21 provenances (voir tableau I)

- 5 répétitions

-21 blocs

- 5 provenances par bloc

- 121 plants par parcelle unitaire tation.

- Deux ou trois lignes de bordures sur le périmètre de la plan-

$$
2232 \text { - Expérience secondaire (P 300-2). }
$$

- Blocs complets

- 10 provenances

- 3 répétitions

- 80 plants par parcelle unitaire

- 2 lignes de bordures sur le périmètre de la plantation. 


\section{3 - Remarques diverses.}

Cette scission en deux expériences s'est révélée nécessaire car le nombre de plants obtenus en pépinière variait beaucoup selon les provenances.

Il convient également de signaler que: 36. 40).

- 3 provenances sont communes aux deux expériences ( 16 ,

- Pour certaines provenances pour lesquelles nous disposions de lots de graines individualisées par arbres-mères $(7,4,2,12)$. les descendants correspondants furent maintenus séparés sur le terrain à l'intérieur des parcelles unitaires affectées à ces provenances.

\section{3 - Mesures et résultats.}

31 - Mesures.

On trouvera sur le tableau 2, la liste des mesures effectuées:

- soit en pépinière,

- soit sur les plantations comparatives.

Une importante mortalité a été provoquée par une gelée tardive très forte $\left(-7^{\circ}\right)$ en mai 1960 , soit six mois après la plantation. Le comptage de survie A 1960 (1) a été suivi par une opération de regarnis, effectuée avec des plants du même âge et de mêmes provenances. Pour les regarnis ultérieurs ( $\Lambda 1961$ et A 1964), on a utilisé des plants d'une autre espèce (Picea abies) pour éviter toute confusion au cours des mesures ultérieures.

Les mesures de débourrement (P 1963) ont porté sur le bourggeon terminal et sur le bourgeon latéral le plus développé du verticille supérieur (2), en empruntant l'échelle de notation suivante:

0 - bourgeon dormant

1 - bourgeon gonflé

2 - aiguilles apparentes dépassant la longueur du bourgeon

3 - aiguilles apparentes commençant à s'écarter à leur extrémité

4 - aiguilles nettement écartées — pousses allongées.

(1) Les lettres $\mathrm{A}$ ou $\mathrm{P}$ avant le millésime désignent la saison (automne ou printemps).

(2) Dans le cas du mélèze, le terme de verticille est impropre, au sens botanique du mot. Cependant, il est utilisé dans cet article pour faciliter la rédaction du texte. Par verticille supérieur, il faut entendre l'ensemble des pousses latérales situées à la base de la pousse terminale. 
TABLEAU II

NATURE ET DATE DES MESURES EFFECTUEES

\begin{tabular}{|c|c|c|c|}
\hline Nature de la mesure & Date & $\begin{array}{c}\text { Ape des plants } \\
\text { (années) }\end{array}$ & Observations \\
\hline Poids de 1000 graines & P 1958 & - & \\
\hline Hauteur & A 1958 & $1-0$ & Classes : $1 \mathrm{~cm}$ \\
\hline Hauteur & A 1959 & $1-1$ & Classes: $5 \mathrm{~cm}$ \\
\hline Survie & A 1960 & 3 & Comptage de tous les plants morts y compris les bordures \\
\hline Survie & A 1961 & 4 & Comptage de tous les plants morts de l'expérience \\
\hline Houteur & A 1961 & 4 & $\begin{array}{l}\text { Classes : } 5 \mathrm{~cm} \\
\text { Echantilion de } 49 \text { emplacements de plants par parcelle untaire }\end{array}$ \\
\hline Survie & P 1963 & 5 & Comptage de tous les plants morts de l'expérience \\
\hline Débourrement & $\begin{array}{l}\text { P } 1963 \\
\text { (9 am } 11 \text { avril) }\end{array}$ & 5 & $\begin{array}{l}\text { Echantillon de } 49 \text { emplacements de plants par parcelle unitaire } \\
\text { Les mesures ont porté sur } \underline{28} \text { parcelles unitaires }\end{array}$ \\
\hline Survie & A 1963 & 6 & $\begin{array}{l}\text { Comptage des plants morts sur un échentillon de } \\
49 \text { emplacements de plants par parcelle unitaire (P } 300-t) \\
36 \text { emplacements (P } 300-2)\end{array}$ \\
\hline Houteur & A 1963 & 6 & $\begin{array}{l}\text { Echantillon de } 49 \text { emplacements de plants par parcelle unitaire } \\
(P 300-1) \text { et de } 36 \text { emplacements (P } 300-2)\end{array}$ \\
\hline
\end{tabular}


32 - Résultats (1).

Tous les résultats seront présentés clans ce chapitre sous forme de graphiques sur lesquels on lira d'abord le numéro des provenances, puis la valeur des moyennes et l'échelle et enfin les différences significatives calculées au seuil $1 \%$ à l'aide du Test de Duncan (à l'exception du cébourrement où le seuil choisi a été $5 \%$ ). Ces différences sont représentées par des traits verticaux, de telle sorte que deux provenances non reliées par un trait vertical sont significativement différentes au seuil utilisé.

\section{1 - Poids de 1000 graines (voir graphique 1).}

Cette mesure a été effectuée selon les règles internationales de l'Association Internationale d'Essais de Semences. Les résultats obtenus confirment ceux présentés par Bouvarel et Lemoine en 1958, à savoir :

- que les graines issues des Alpes françaises sont nettement plus lourdes (8,40 à $10,38 \mathrm{~g}$ pour 1000 graines),

- que les graines provenant de Pologne et des Sudètes se révèlent plus légères (3,05 à 4,36 g pour 1000 graines),

- que les graines des Tatras et des Alpes (autres que françaises) semblent légèrement plus lourdes que celles des Sudètes et qu'elles se distinguent significativement de celle des Alpes françaises,

- qu'en ce qui concerne les graines de provenance artificielle ou d'hybride, aucune règle générale ne peut être dégagée sauf qu'elles sont moins lourdes que celles des provenances des Alpes françaises.

- qu'il n'apparaît pas de liaison rigoureuse, pour une région donnée, entre le poids de 1000 graines et l'altitude.

\section{2 - Survie.}

\section{1 - Mesure $1960 \mathrm{~A}$.}

La très forte gelée tardive survenue en mai 1960 a provoqué des mortalités importantes qui masquent probablement l'aptitude normale à la survie des différentes provenances. En effet, au moment de cette chute de température tous les sujets étaient débourrés.

Par ailleurs, quelques provenances installées dans des trous à gelée mouilleux se sont trouvées pénalisées (provenances 40 dans l'expérience P 300-1 et 16 dans P 300-2).

(1) La Station de Biométrie du C.N.R.F, a participé à l'exécution des calculs. 


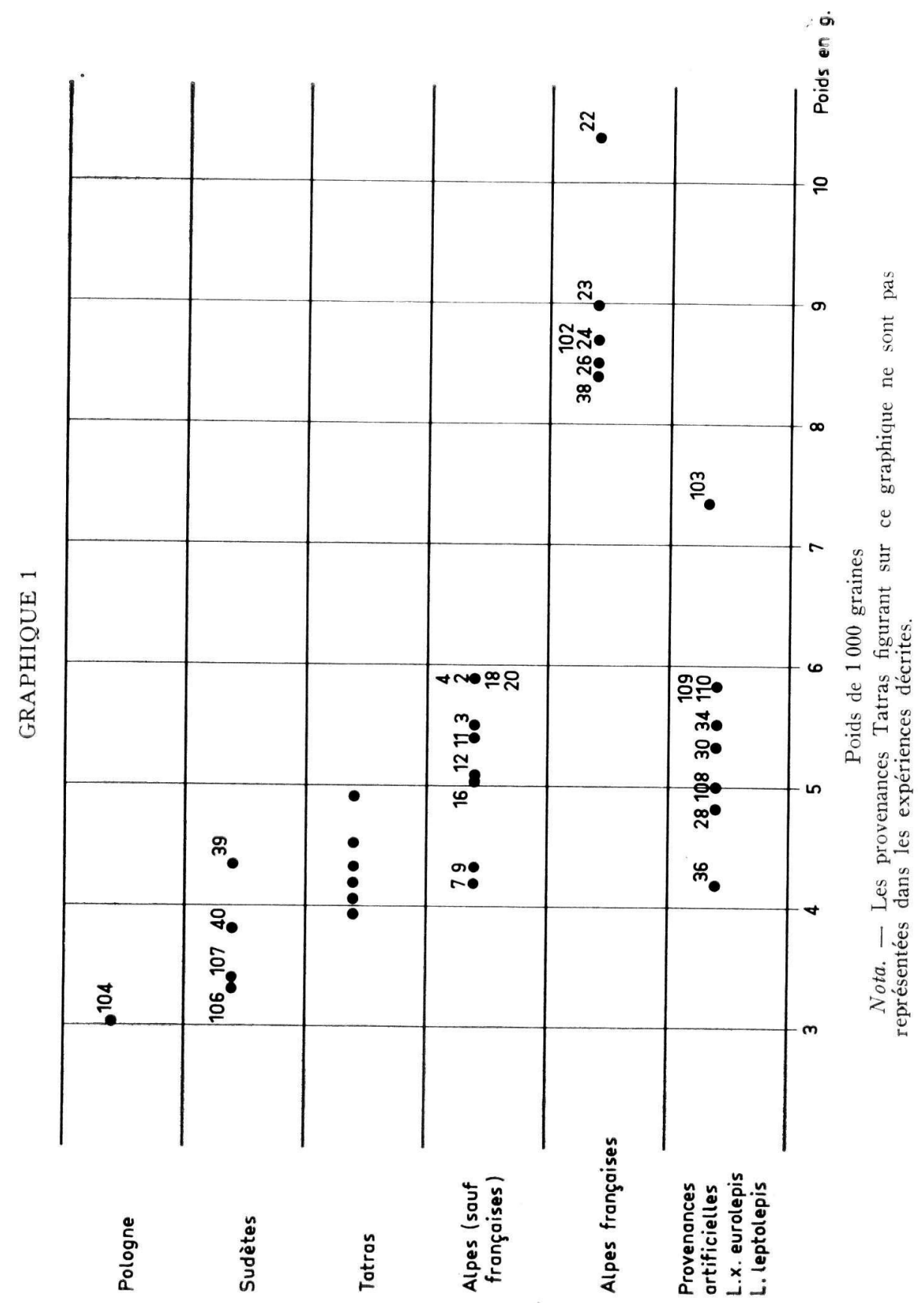


On trouvera sur le graphique 2 le classement obtenu pour les deux expériences.

L'unique lot de mélèze du Japon et les provenances des Alpes françaises présentent une forte mortalité. Au contraire, les provenances 3 et 12 (Autriche) et 104 (Pologne) se situent en tête du classement de survie. On remarque que les différences significatives calculées se révèlent assez faibles.

\section{2 - Mesure $1961 \mathrm{~A}$.}

Les résultats sont présentés également sur le graphique 2 .

Une opération de regarnis a été effectuée à l'automne 1960. La mortalité enregistrée à l'automne 1961 exprine mieux l'aptitude à la reprise que la mesure précédente. Il faut tenir compte cependant du fait que les plants utilisés pour les regarnis étaient tous stockés (sauf le lot mélèze du Japon - 36-) à proximité des plantations comparatives et avaient subi l'action affaiblissante des gelées de mai 1960.

Si l'on rapproche les classements de 1960 A et 1961 A, on enregistre pour ce dernier, une amélioration sensible de la position du mélèze du Japon, des hybrides et d'une provenance des Sudètes (40).

\section{3 - Hanteur.}

\section{1 - Pépinière.}

Les classements sont présentés sur le graphique 3 (hauteur des semis 1-0 et des plants 1-1).

Les hauteurs des semis et celles des plants ne se présentent pas dans le même ordre. Pour les plants, les hybrides danois (110 et 109 ) et les provenances des Sudètes se classent très nettement au premier rang. Les provenances alpines françaises manifestent à ce stade une croissance beancoup plus faible alors que sous la forme de semis elles n'étaient pas sensiblement différentes des autres provenances.

\section{2 - Hauteur 1961 A et 1963 A.}

Aux âges (calculés depuis le semis) de 4 et 6 ans, les résultats enregistrés pour les plants de 2 ans semblent se confirmer (voir graphique 4).

Nous n'avons pas jusqu'à présent fourni le détail des calculs de variance. A titre d'exemple, on trouvera ci-dessous les tableaux d'analyse correspondant aux mesures de hauteur 1963 A pour P 300-1 et P 300-2. 
GRAPHIQUE 2

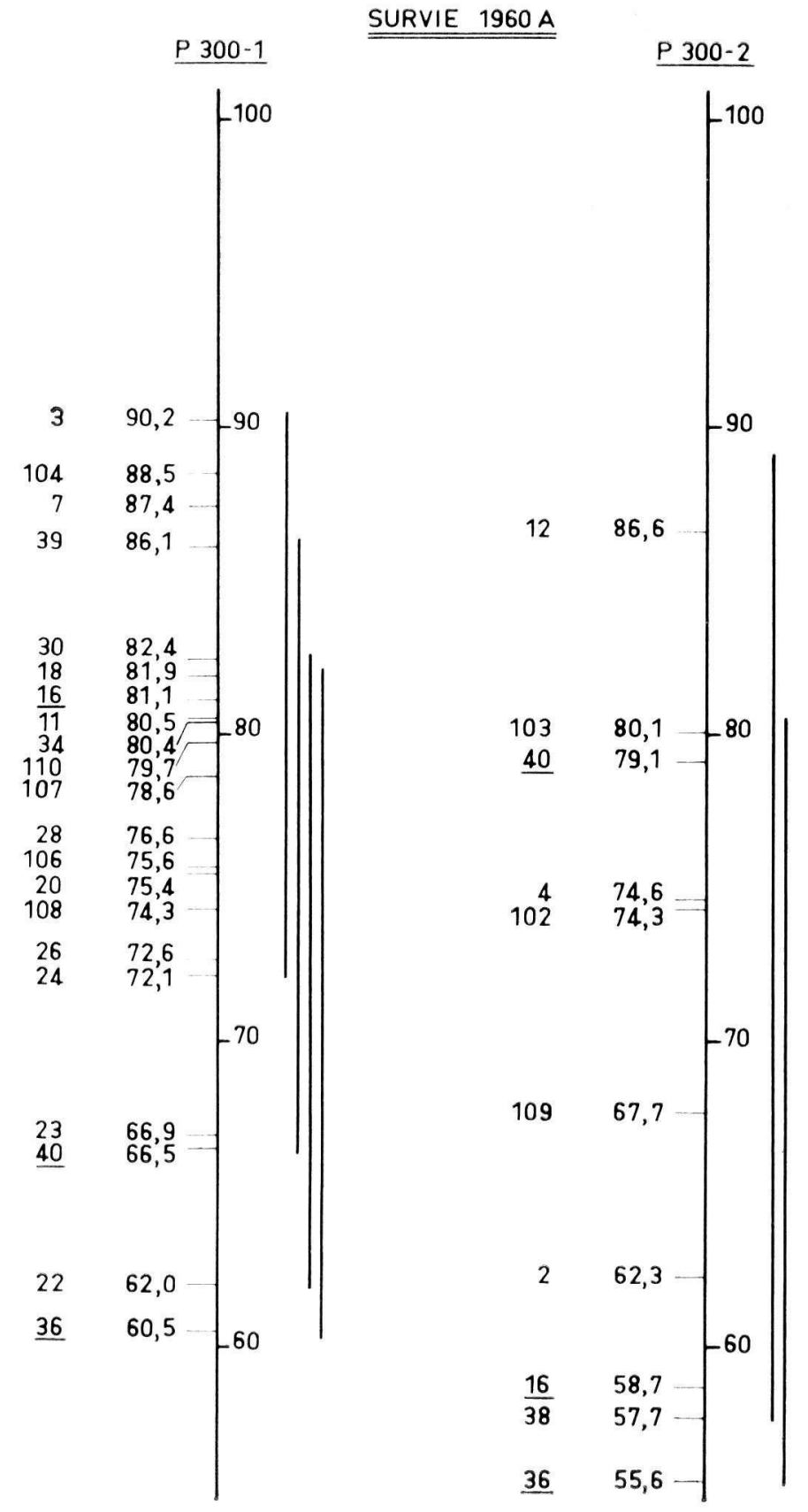


GRAPHIQUE 2

SURVIE 1961A

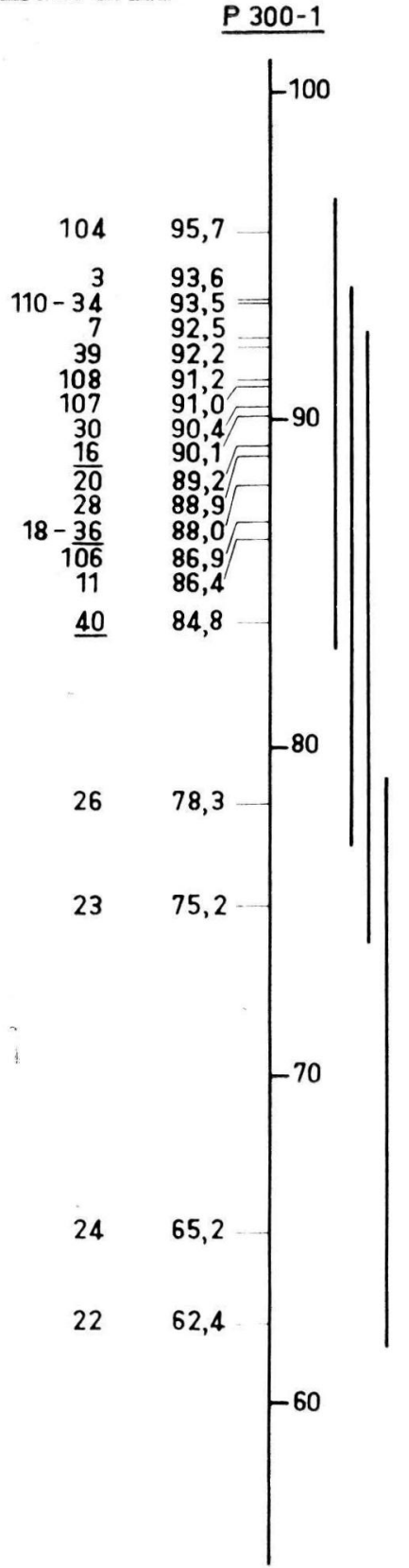

P 300-2

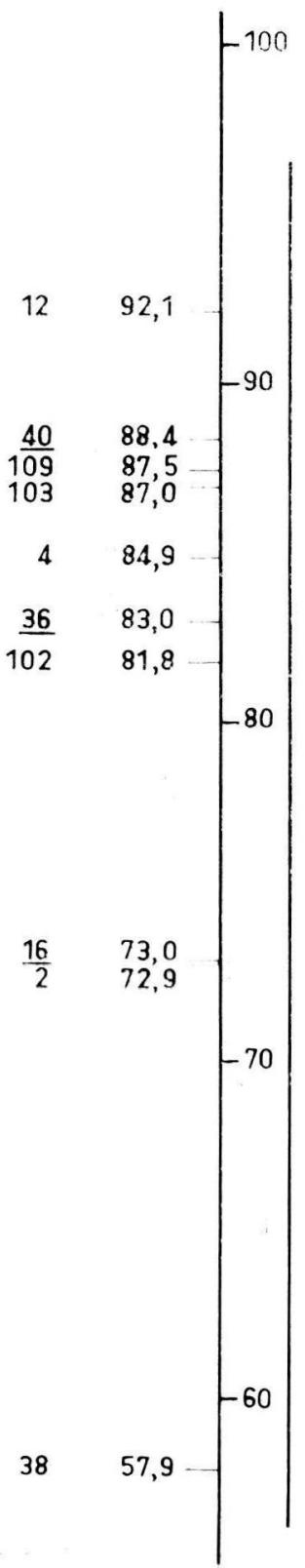




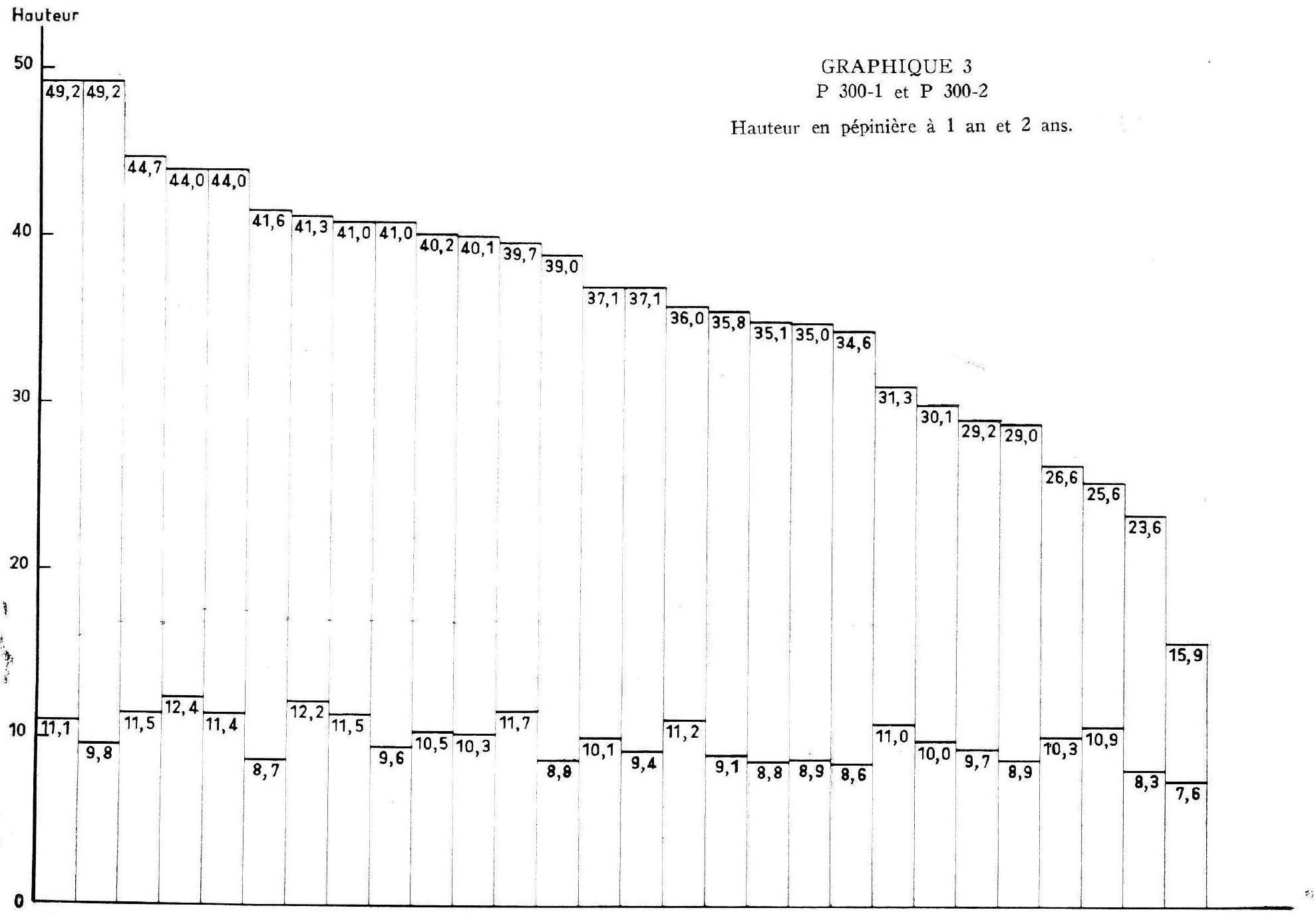


GRAPHIQUE 4

\section{HAUTEUR 1961A}

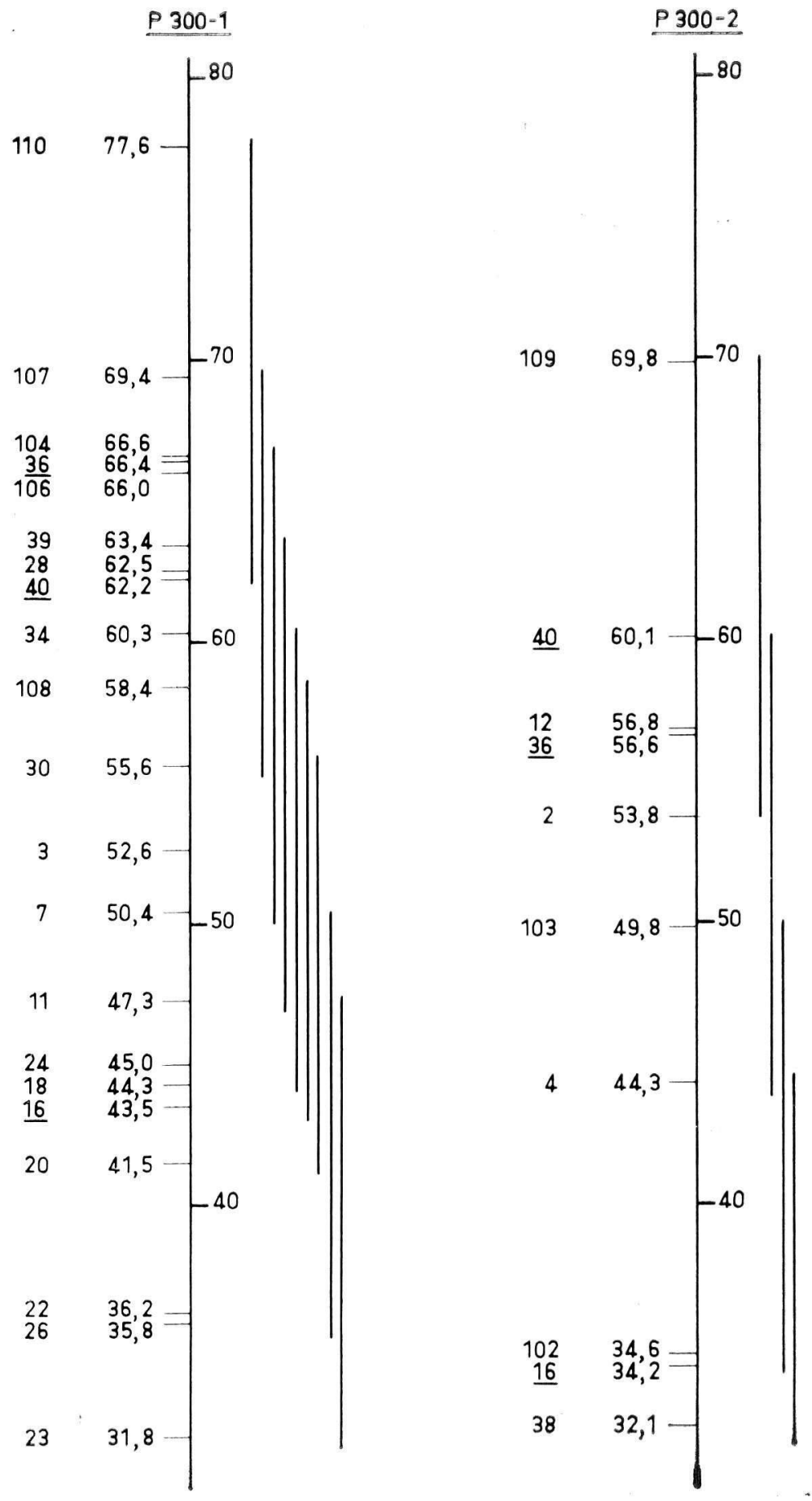


GRAPHIQUE 4

\section{HAUTEUR 1963A}
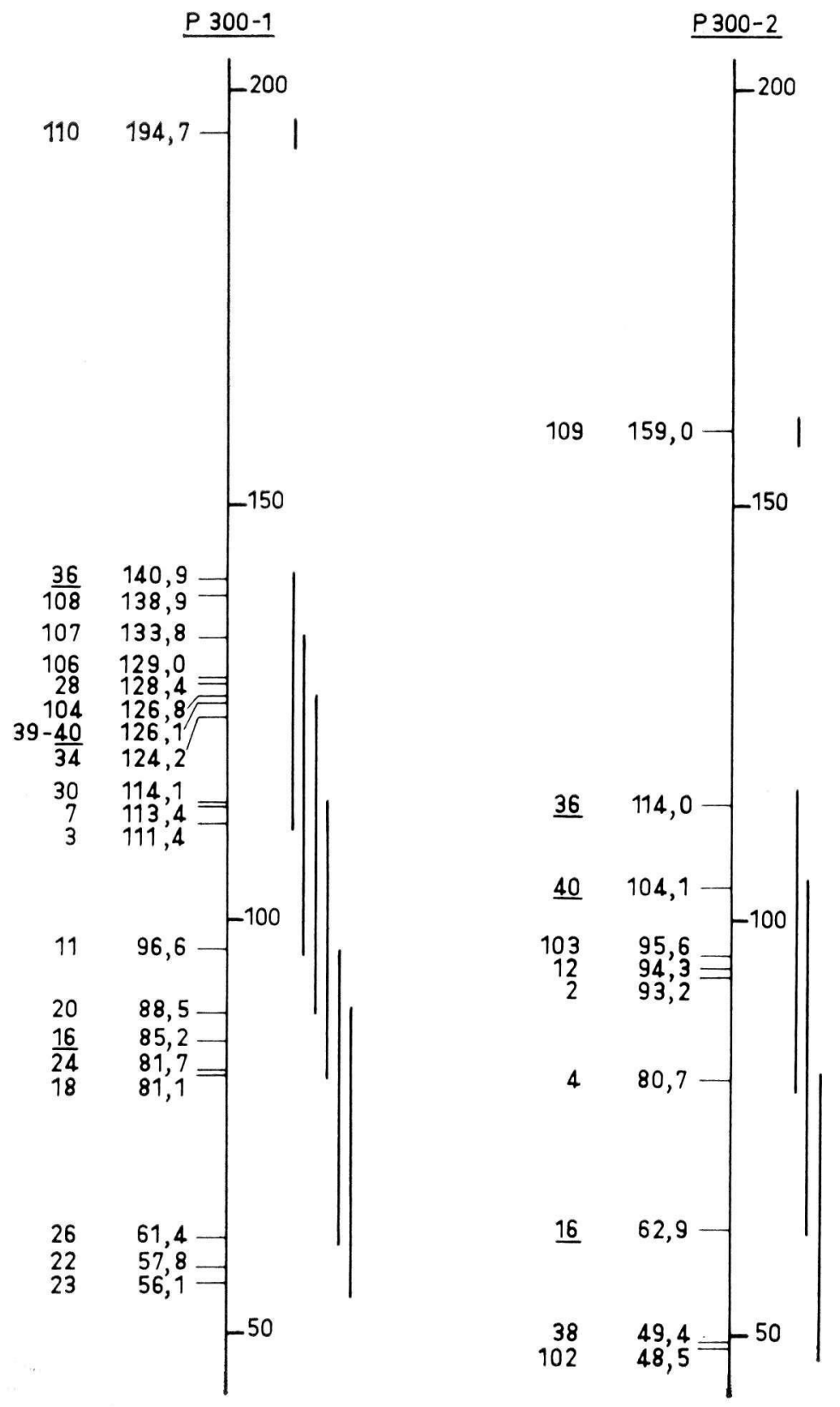
P $300-1$

\begin{tabular}{|c|c|c|c|}
\hline Origine de la variation & $\begin{array}{c}\text { Somme des } \\
\text { carrés }\end{array}$ & $\begin{array}{c}\text { Degré } \\
\text { de } \\
\text { liberté }\end{array}$ & Carré moyen \\
\hline Blocs (provenances ignorées) & $48 \propto 25,05$ & 20 & \\
\hline Provenances (blocs éliminés) & 93087,84 & 20 & $E_{t}=4654,392$ \\
\hline Blocs (provenances éliminées) & 26735,14 & 20 & $E_{b}=1336,757$ \\
\hline Provenances (blocs ignorés) & 114377,75 & 20 & \\
\hline Erreur & 23. 332,98 & 64 & $E_{e}=364,578$ \\
\hline Total & 164445,87 & 104 & \\
\hline
\end{tabular}

Le test $\mathrm{F}$ de Snedecor indique que les provenances sont significativement différentes: $\mathrm{F}=\frac{\mathrm{Et}}{\mathrm{Ee}}=12,8(2,25$ au seuil de $1 \%)$.

Si t est le nombre de provenances et $\mathrm{k}$ le nombre de provenances par bloc, le facteur de pondération s'écrit:

$$
\dot{\omega}=\frac{\mathrm{Eb}-\mathrm{Ee}}{\mathrm{t}(\mathrm{k}-1) \mathrm{Eb}}=0,00866
$$

La variance d'un total ajusté est donc:

$$
\mathrm{s}_{\mathrm{t}}{ }^{2}=5[1+(\mathrm{t}-\mathrm{k}) \hat{\omega}] \mathrm{Ee}=2075,4227
$$

et l'écart-type: $s_{\mathrm{t}}=45,56$.

L'écart-type d'une moyenne ajustée de 5 mesures est:

P 300-2

$$
\mathrm{s}_{\mathrm{t}}^{-}=9,062 \text {. }
$$

\begin{tabular}{|l|r|r|r|c|}
\hline \multicolumn{1}{|c|}{$\begin{array}{c}\text { Origine de la } \\
\text { variation }\end{array}$} & $\begin{array}{c}\text { Somme des } \\
\text { carrés }\end{array}$ & $\begin{array}{c}\text { Degré de } \\
\text { liberté }\end{array}$ & Carré moyen & $F$ \\
\hline Blocs & 437,24 & 2 & 218,62 & \\
Traitements & 29377,67 & 9 & 3264,86 & $11,43 \times \times$ \\
Erreur & $\underline{5142,06}$ & $\underline{18}$ & 285,67 & \\
Total & 34956,97 & 29 & & \\
\hline
\end{tabular}

$\mathrm{x} \times$ : significatif au seuil de $1 \%$. 
L'écart-type d'une moyenne est: $\mathrm{s}_{\mathrm{t}}=9,758$.

Les différences significatives ont été calculées en appliquant le test à ranges multiples de Duncan au seuil de $1 \%$.

\begin{tabular}{|c|c|c|c|c|c|c|c|c|c|c|}
\hline Expérience & 2 & 3 & 4 & 5 & 6 & 7 & 8 & 9 & 10 & 12 \\
\hline P $300-1$ & 34,07 & 35,52 & 36,52 & 37,34 & 37,79 & 38,33 & 38,69 & 39,06 & 39,33 & 39,78 \\
P $300-2$ & 39,72 & 41,67 & 42,74 & 43,52 & 44,20 & 44,79 & 45,28 & 45,67 & 45,96 & 46,45 \\
\hline
\end{tabular}

On remarquera en particulier, que les hybrides danois sont significativement supérieurs à tous les autres lots. Le mélèze du Japon (36) est remonté dans le classement.

Les observateurs ont été frappés par la différence de variabilité entre provenances en ce qui concerne le caractère croissance en hauteur.

Nous avons procédé au calcul des coefficients de variation pour chaque parcelle unitaire (le pourcentage de l'écart-type par rapport à la moyenne); nous avons ensuite affecté à chaque provenance à l'intérieur de chaque bloc, une note allant de 0 à 4 , en fonction du rang de classement des coefficients de variation (note 0 pour le coefficient le plus élevé, note 4 pour le coefficient le plus faible). Comme il y a 5 répétitions, la note globale de chaque provenance est donc comprise entre 0 et 20 .

Le résultat de cette notation se présente comme suit:

$\begin{array}{ll}\text { Note } & \text { Provenances } \\ - & - \\ 19 & 110 \\ 15 & 104 \\ 14 & 107-36 \\ 12 & 7-34-39 \\ 10 & 11-30 \\ 9 & 3-16-24-40-106 \\ 8 & 23-28-108 \\ 7 & 26 \\ 6 & 18-20 \\ 5 & 22\end{array}$

On constate que l'hybride danois se révèle nettement plus homogène que tous les autres lots. Par contre, les provenances alpines sont généralement plus hétérogènes. 
Pour ce caractère, les provenances des Sudètes apparaissent moins groupées que pour la croissance en hauteur et comme nous le verrons, le débourrement.

\section{4 - Débourrement.}

3241 - Bourgeon latéral, le plus développé du verticille supérieur.

Les observations n'ont porté que sur 28 parcelles unitaires. On a calculé la moyenne des notes pour chacune de ces parcelles et l'analyse de variance a donc été effectuée avec un nombre inégal de répétitions par provenance. Les différences significatives indiquées correspondent au seuil de $5 \%$.

L'hybride danois (110), la provenance polonaise et celle des Sudètes se situent dans le groupe le plus tardif. Au contraire, les mélèzes alpins se révèlent les plus précoces, ainsi que le mélèze du Japon (voir graphique 5).

\section{2 - Bourgeon terminal.}

L'analyse a concerné le pourcentage de bourgeons dormants (stade 0 ) et les calculs ont été conduits comme précédemment après transformation des pourcentages en arc sinus $\sqrt{\mathrm{P} \text {. }}$

L'hybride danois au moment de la mesure (avril 1963) avait encore une très forte proportion (92\%) de bourgeons terminaux dormants (il est à ce point de vue significativement différent de tous les autres lots).

Il existe, en gros, une analogie certaine avec le classement obtenu précédemment, mais il faut signaler au moins une différence concernant le mélèze du Japon qui a une proportion de bourgeons terminaux dormants plus forte que ne le laissent prévoir les résultats des notations sur le verticille supérieur.

\section{4 - Interprétation des résultats.}

Les résultats précédents nous ont suggéré deux séries d'interprétation :

- étude de liaisons éventuelles entre différentes mesures,

- essai de regroupement des provenances en fonction de leur comportement.

\section{1 - Liaisons entre les différentes mesures.}

411 - Hauteur et survie.

Il n'a pas été possible de mettre en évidence sur l'ensemble des lots, une corrélation significative entre les moyennes de hauteur et 
GRAPHIQUE 5

\section{DEBOURREMENT 1963P \\ P $300-1$}

Verticille supérieur

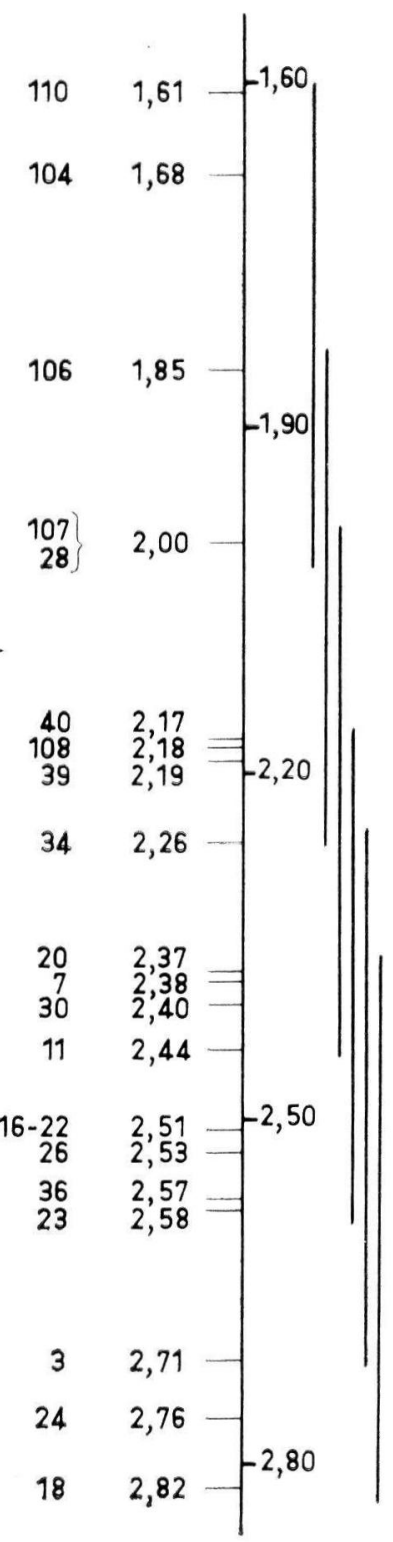

Bourgeon terminal

110<smiles>CC(=O)C(C)C(=O)O</smiles>

104 106

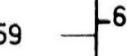

40

11

3

$$
20
$$

16
23
24
7

18

22 
de survie. Néanmoins, les provenances alpines françaises présentent à la fois une mauvaise survie et une faible croissance en hauteur. Au contraire, l'hybride danois se situe en tête du classement pour les deux caractères.

412 - Liaisons entre les hauteurs à différents âges.

Les corrélations calculées sont résumées dans le tableau ci-dessous :

\begin{tabular}{|c|c|c|}
\hline Mesures étudiées & $\begin{array}{c}\text { Coefficients } \\
\text { de } \\
\text { corrélation }\end{array}$ & Seuil de signification \\
\hline Hauteur 1-0 et hauteur 1-1 & 0,441 & pas significatif à $5 \%$ \\
Hauteur 1-1 et hauteur à 4 ans & 0,823 & significatif à 1 \% \\
Hauteur 1-1 et hauteur à 6 ans & 0,904 & significatif à $1 \%$ \\
\hline
\end{tabular}

Dans cette analyse, seules les provenances de mélèze d'Europe ont été prises en considération car les lots d'hybrides et le mélèze du Japon se comportent trop différemment des mélèzes d'Europe, en ce qui concerne la croissance juvénile. On trouvera une autre présentation de ces résultats sur le graphique 6 .

Il apparait donc que pour les mélèzes d'Europe:

- le classement de hauteur obtenu à un an se révèle peu intéressant; à ce stade, il y a probablement interférence entre la capacité de croissance propre aux provenances (génotype de la population) et l'action due au poids des graines (réserves de l'endosperme élevées, par exemple pour les provenances alpines françaises).

- le classement de hauteur obtenu à deux ans, donc à un stade encore relativement juvénile, reste valable 4 ans après. L'action perturbatrice due à l'installation des plants sur le terrain peut expliquer que le coefficient soit plus faible à 4 ans qu'à 6 ans. Cette action tendrait à s'atténuer avec le temps.

Si ce phénomène devait se confirmer au cours des prochaines années - ce qui est une hypothèse vraisemblable - l'étude de la variabilité infraspécifique de cette espèce pour le caractère de la croissance en hauteur, pourrait être réalisée dans le cadre de tests précoces d'une clurée minimum de 2 ans.

\section{3 - Hauteur et débourrement.}

La liaison entre ces deux caractères a été abordée à deux niveaux :

- celui des provenances,

- celui des individus à l'intérieur de chaque provenance. 
GRAPHIQUE 6

P $300-1$

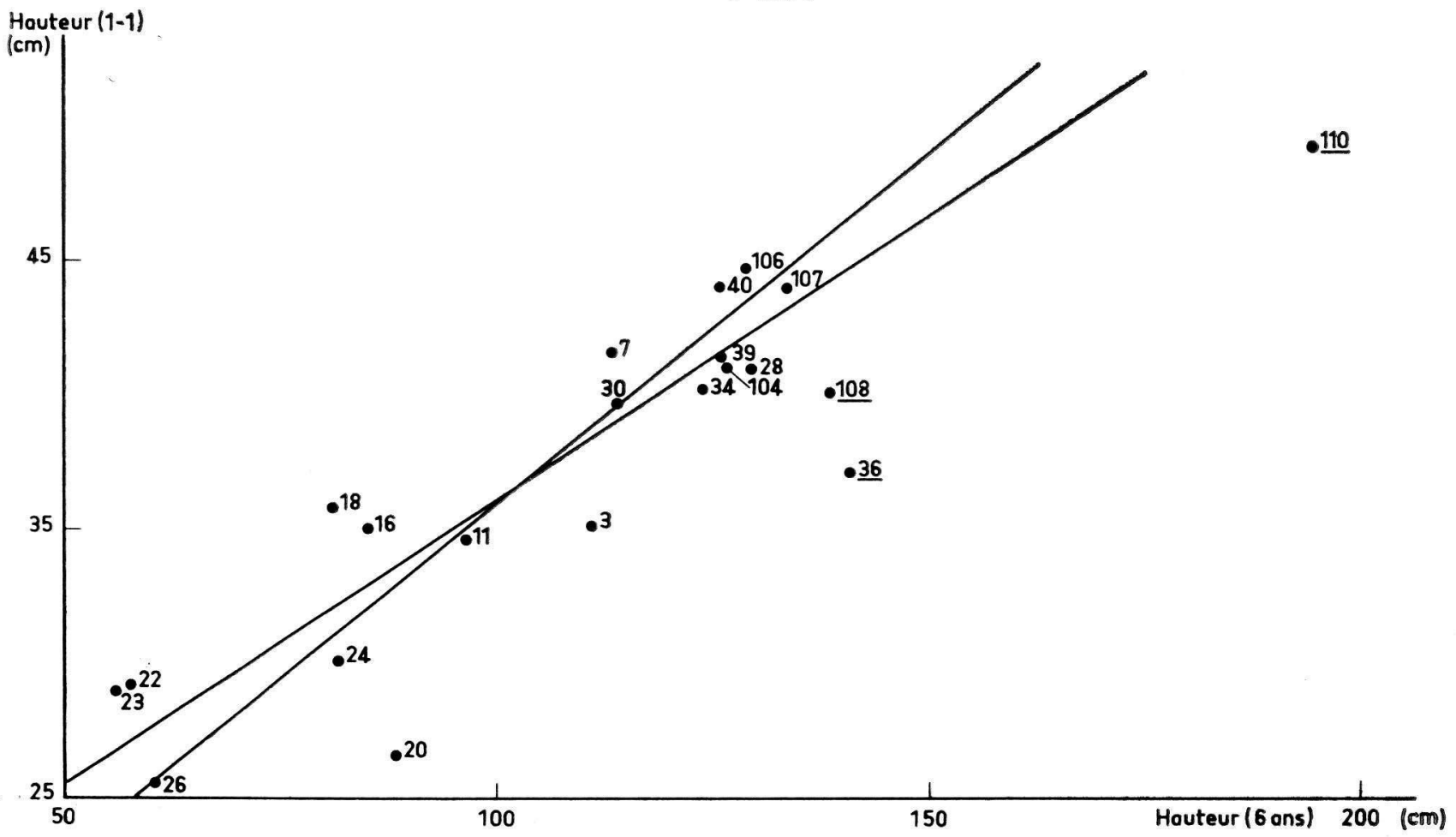

Les numéros soulignés ne sont pas entrés en compte pour le calcul de la corrélation.

Liaison entre la hauteur à 2 ans et à 6 ans. 
4131 - Corrélation au niveau des provenances.

Le coefficient de corrélation entre les moyennes ajustées des hauteurs à 6 ans et le pourcentage (transformé en arc sinus $\sqrt{\mathrm{P}}$ ) de plants à bourgeons terminaux non débourrés en avril 1963 est de $+0,882$, significativement différent de 0 au seuil de $1 \%$ (voir graphique 7). Ce coefficient a été calculé en tenant compte de tous les lots inclus dans l'expérience P 300-1.

Il s'avère donc que les populations à débourrement tardif présentent les meilleures croissances. Ce phénomène peut être dî̀, au moins partiellement, au fait que les populations à forte fréquence de plants précoces subissent des dégâts plus importants du fait des

TABLEAU 3

Corrélation hauteur-débourrement au niveau individuel.

\begin{tabular}{|c|c|c|c|c|}
\hline $\begin{array}{l}\text { Provenances } \\
\text { (1) }\end{array}$ & Blocs & $\begin{array}{c}\text { Nombre } \\
\text { d'observations }\end{array}$ & $\begin{array}{l}\text { Coefficients } \\
\text { de corrélation }\end{array}$ & $\begin{array}{l}\text { Seruil de sigaiflcation } \\
\text { (2) }\end{array}$ \\
\hline $\begin{array}{r}110 \\
36 \\
106 \\
107 \\
106 \\
106 \\
28 \\
28 \\
28 \\
104 \\
39 \\
39 \\
40 \\
34 \\
30 \\
30 \\
7 \\
3 \\
11 \\
20 \\
16 \\
16 \\
16 \\
24 \\
18 \\
18 \\
26 \\
22 \\
23\end{array}$ & $\begin{array}{r}5 \\
1 \\
3 \\
5 \\
7 \\
9 \\
1 \\
5 \\
9 \\
9 \\
3 \\
9 \\
21 \\
7 \\
1 \\
7 \\
3 \\
11 \\
7 \\
5 \\
3 \\
5 \\
7 \\
13 \\
1 \\
3 \\
9 \\
15 \\
15\end{array}$ & $\begin{array}{l}49 \\
39 \\
48 \\
46 \\
42 \\
39 \\
49 \\
42 \\
44 \\
47 \\
47 \\
47 \\
38 \\
42 \\
41 \\
43 \\
47 \\
40 \\
41 \\
42 \\
31 \\
34 \\
39 \\
39 \\
40 \\
43 \\
42 \\
30 \\
31\end{array}$ & $\begin{array}{l}-0,45 \\
-0,11 \\
-0,39 \\
-0,47 \\
-0,48 \\
-0,49 \\
-0,27 \\
-0,52 \\
-0,28 \\
-0,41 \\
-0,56 \\
-0,68 \\
-0,36 \\
-0,19 \\
-0,61 \\
-0,35 \\
-0,54 \\
-0,53 \\
-0,31 \\
-0,40 \\
-0,56 \\
-0,20 \\
-0,27 \\
-0,54 \\
-0,58 \\
-0,41 \\
-0,32 \\
-0,50 \\
-0,24\end{array}$ & 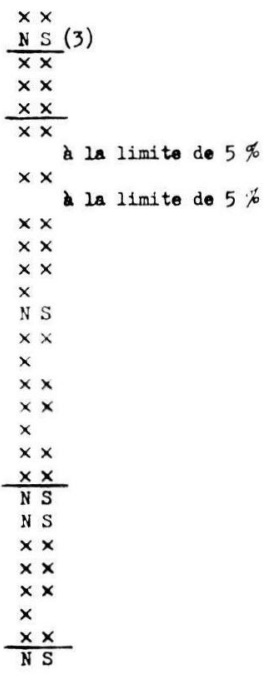 \\
\hline
\end{tabular}

$\mathrm{xx}$ : significativement différent de 0 au seuil de $1 \%$ $\mathrm{x}$ : significativement différent de 0 au seuil de $5 \%$

N S : non significatif

(1) Les provenances sont présentées dans l'ordre décroissant des moyennes ajustées de hauteur à 6 ans.

(2) Le seuil indiqué est présenté avec réserves lorsque les distributions marginales ne sont pas normales.

(3) Les résultats soulignés ont seuls des distributions marginales normales pour le débourrement. 


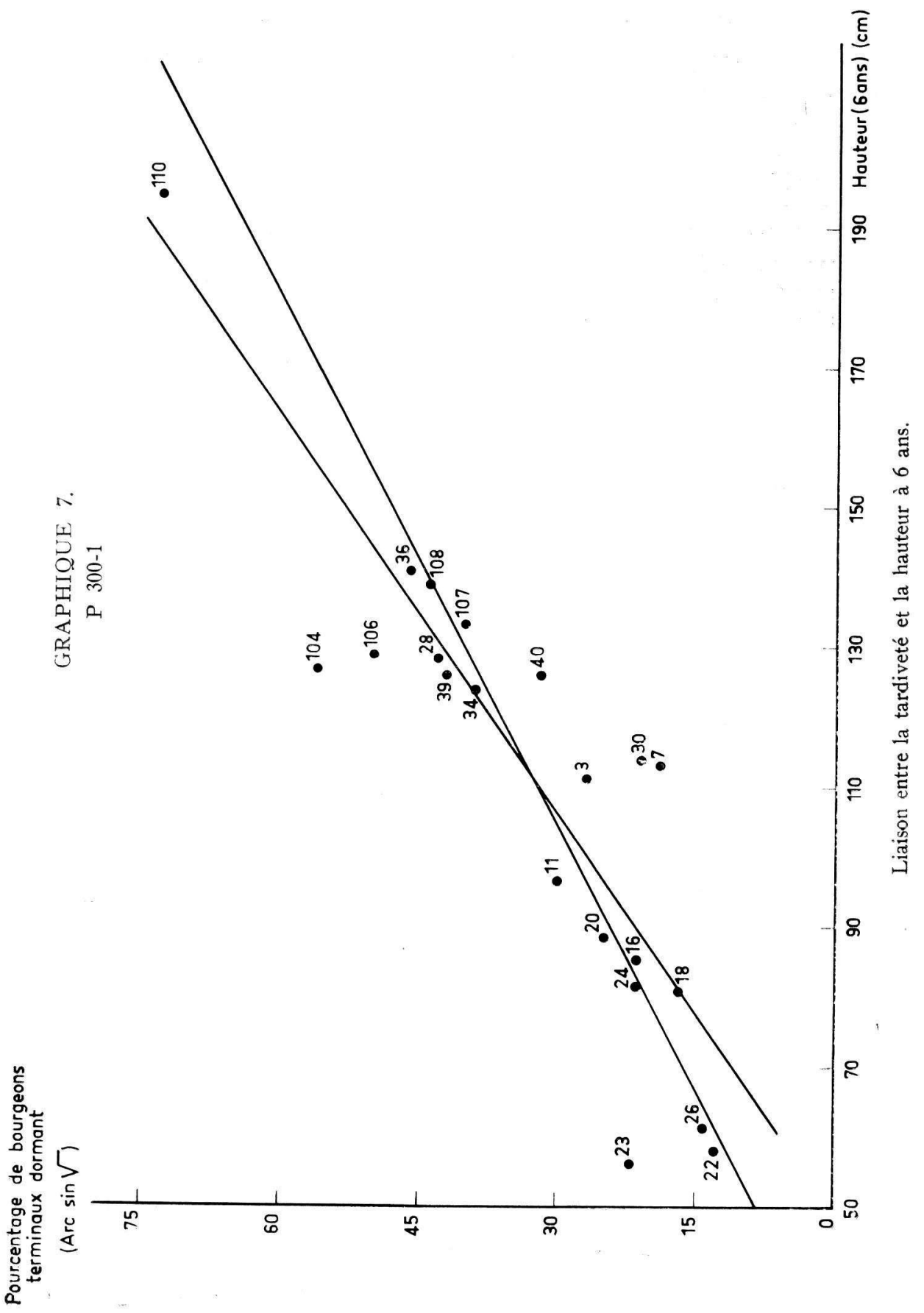


gelées tardives. Il faut cependant signaler que la première gelée de mai 1960 est intervenue alors que tous les plants - même les plus tardifs - avaient commencé leur croissance en hatteur. Cette gelée aurait dî pénaliser toutes les provenances de façon à peu près équivalente.

\section{2 - Corrélation au niveau individuel.}

Un calcul du même genre a été fait pour chaque parcelle unitaire objet d'observations de débourrement en avril 1963 et at1 niveau des individus.

Les caractères pris en considération sont respectivement:

- pour le débourrement: la somme des notations concernant le bourgeon terminal et le bourgeon latéral choisi sur le verticille supérieur (échelle de notations de 0 à 7 ).

- pour la hauteur: la mesure de $1963 \mathrm{~A}$.

Les résultats présentés sur le tableau 3 appellent les observations suivantes:

Dans la plupart des cas, les distributions marginales des notations de débourrement ne sont pas normales. Il faut donc émettre des réserves sur les seuils de signification proposés.

On remarquera néanmoins que les coefficients sont toujours négatifs et que la liaison paraît très forte dans 17 cas sur les 29 étudiés. Le mélèze du Japon correspond au coefficient le plus faible (en valeur absolue). L'hypothèse que nous cherchons à vérifier ne s'appliquerait donc pas à cette espèce. Pour un certain nombre de parcelles de provenances alpines $(16,23)$ la liaison apparente est variable selon les parcelles. Fn fait, celles-ci ont subi de fortes mortalités et l'on a pu vérifier, notamment en comparant les notations de débourrement d'avril 1963 aux mesures de survie de A 1963 , que ce sont généralement les plants les plus précoces qui ont disparu. Par ailleurs, ces plants les plus précoces se classaient en A 1961 parmi les plus petits de chaque parcelle unitaire. Ceci a pu contribuer à réduire la valeur du coefficient de corrélation que l'on aurait dû trouver en absence de mortalité.

Une provenance artificielle (34) se comporte de façon aberrante sans que l'on puisse fournir d'explication.

Pour les provenances des Sudètes et de Pologne (104, 107, 106, 39 ) et les hybrides $(110,108)$, les résultats sont homogènes avec une corrélation apparente élevée.

\section{3 - Conclusion.}

Il s'avère intéressant de rapprocher ces deux types de résultats. Au niveau des populations, il est possible de mettre en évidence une forte corrélation positive entre les moyennes de hauteur et la 
proportion de bourgeons terminaux non débourrés donc la tardiveté. Au niveau des individus, pour chaque provenance, il apparait une certaine corrélation moins sûre, négative entre la hauteur et la précocité, ce qui rejoint la première conclusion.

Si de telles hypothèses se confirmaient d'une part sur le dispositif expérimental étudié au cours des prochaines années, d'autre part sur les dispositifs installés dans d'autres pays, nous disposerions d'un caractère de sélection précoce fort utile.

\section{2 - Essai de regroupement des provenances en fonction de leur comportement.}

Un essai de groupement géographique des provenances des deux expériences en fonction des hauteurs 1963 A est présenté sur le graphique 8 en prenant la base 100 pour les deux hybrides danois. Cela est possible, car le rapport des hauteurs des trois témoins à celui des deux hybrides donne des valeurs très voisines.

\section{Groupe $I$ - Hybrides danois (F1).}

Les deux lots ont un comportement presque identique. Leur croissance est excellente dès la pépinière (significativement supérieure à celle de tous les autres lots en 1963) aussi bien dans les zones fertiles que dans celles où les conditions se révèlent moins bonnes (en particulier stations mouilleuses). On a ici une confirmation du fait bien connu suivant: la variation phénotypique d'une population F1 est généralement faible. Leur survie est également très bonne. Le lot 110 se caractérise enfin par un fort pourcentage de sujets tardifs (il n'y a pas eu d'observation de précocité du lot 109).

\section{Groupe II - Mélèze du Japon et hybride de Dunkeld ou back- cross (F2).}

L'hybride de Dunkeld se différencie très peu du mélèze du Japon. Classés après les mélèzes des Sudètes en pépinière, ces deux lots ont manifesté depuis une croissance en hauteur supérieure à ces derniers. On reconnaît la croissance initiale plus rapicte du mélèze du Japon.

Ia gelée tardive de mai 1960 a particulièrement touché le mélèze du Japon qui a perdu environ $40 \%$ de plants dans les deux expériences.

Cette sensibilité a d'ailleurs été confirmée par une plantation voisine de mélèze du Japon détruite à $75 \%$. La reprise des plants, remplaçant les morts, a d'ailleurs été satisfaisante; le mélèze du Japon est nettement mieux classé après les observations de survie de l'automne 1961. 
Groupe 111 - Sudites et Pologne.

(On est frappé par la très grande homogénéité des résultats des quatre lots provenant des Sudètes. Le lot polonais dont le comportement en matière de croissance semble peu différent a été rattaché à ce groupe.

Ces provenances se caractérisent par:

- de petites graines,

- une survie correcte, excellente pour le mélèze polonais, rope,

- une croissance excellente, la meilleure pour les mélèzes d’Eu-

- un débourrement tardif: ce phénomène est encore plus accusé pour le lot polonais.

\section{Groupe IV - Provenances artificielles.}

I.es provenances artificielles se classent dans un groupe relativement homogène bien qu'elles se situent dans des régions géographiques très éloignées. En ce qui concerne les trois caractères: survie, débourrement et croissance, les provenances Neumunster et surtout Schlitz se comportent aussi bien que le matériel des Sudètes.

\section{Groupe $V$ - Alpes du Nord, Intérieures et Tyrol.}

La délimitation de ce groupe est sans doute contestable, car il couvre une zone trop étendue de l'aire du mélèze. L’imprécision provient d'un échantillonnage insufffisant de provenances. La survic de ces provenances est satisfaisante dans l'ensemble.

En ce qui concerne la croissance, les résultats s'avèrent moins favorables et plus hétérogènes que pour les groupes précédents (bien que les différences avec les groupes III et IV ne soient pas significatives, au seuil de $1 \%$ ). La provenance 11 du Sud-Est des Alpes et 4 des Alpes centrales du Nord se situent dans le bas du classement. Les provenances de ce groupe, dont le débourrement a été mesuré, se classent parmi les plus précoces.

\section{Groupe VI - Alpes méridionales (Italie).}

Si le comportement à la reprise de ces provenances se différencie peu de celui des groupes précédents, elles accusent une croissance en hatuteur significativement inférieure à celles des mélèzes des Sudètes dont elles se distinguent également par leur précocité.

Dans ce groupe, la provenance Cavedine (20) apparaît comme la meilleure. 


\section{Groupe VII - Alpes occidentales (France).}

Toutes les provenances classées dans ce groupe ont un comportement inférieur au point de vue survie et croissance, avec une précocité significativement supérieure.

Il est possible de les distinguer de toutes les autres provenances par la grosseur de leur graine (poids de 1000 graines: entre $8,4 \mathrm{~g}$ et $10,4 \mathrm{~g})$.

Dans ce groupe, la provenance briançonnaise Montgenèvre (24) près de la frontière italienne, serait la moins mauvaise.

Cette classification n'a qu'un caractère indicatif car l'échantillonnage de provenances expérimentées est un peu trop inégal selon les régions. Par ailleurs, aucun mélèze des Tatras n’y figure.

\section{5 - Choix des sources de graines pour le reboisement.}

L.e rôle essentiel de ce type d'expérimentation consiste à indiquer aux reboiseurs les sources de graines auxquelles ils doivent faire appel pour obtenir le meilleur classement.

Dans le cas particulier, il convient d'émettre au moins deux réserves :

- les résultats obtenus concernent un matériel encore jeune (6 ans), même si l'on peut penser que les classements de hauteur et précocité resteront à peu près identiques dans l'avenir, il n'est pas possible actuellement d'émettre un avis sur deux caractères importants. à savoir la forme et la résistance à la pezize du mélèze. Des chercheurs étrangers, par exemple, ont révélé que certaines provenances polonaises présentaient une forme défectueuse. Enfin, un jugement définitif concernant la croissance ne pourra être établi qu'au moment où il sera possible d'effectuer des mesures de volume. c'est-à-dire dans un délai d'une dizaine d'années.

- ces résultats concernent une région très océanique, et de basse altitude. Les réactions de ces provenances pourraient être différentes dans un autre milieu. Nos conclusions devront done être comparées avec les résultats obtenus par les autres participants à l'expérience internationale. sur des plantations situées en climat plus continental.

Tenant compte de ces réserves, il est possible de recommander aux reboiseurs désirant planter du mélèze à basse ou moyenne altitude, en climat atlantique par exemple on Bretagne, Normandie, ou dans l'ouest $d u$ Massif Central, d'utiliser soit des graines d'hybride danois, soit des graines récoltées dans les Sudètes.

Le verger d'Humlebaeck produit annuellement une trentaine de kilogrammes de graines d'hybride et grâce à l'amabilité du Dr BAR- 
NER (1), l'Administration des Eaux et Forêts pourrait peut-être acquérir une partie de cette récolte. Mais cette source se révélerait probablement insuffisante, aussi apparait-il souhaitable d'établir en France un verger à graines à partir de clones sélectionnés par les Danois.

La source Sudètes est maintenant disponible; le service forestier tchèque organise des récoltes sur des peuplements porte-graines sélectionnés.

Les graines récoltées dans les peuplements naturels français devraient être formellement prohibées pour ce genre de reboisement. $\mathrm{Ce}$ fait est peut-être connu depuis longtemps, mais il ne semble pas inutile de le rappeler.

En ce qui concerne les autres sources alpines. Suisse, Autriche, Italie, les résultats assez irréguliers enregistrés ne permettent pas de se prononcer de façon définitive; mais, compte tenu de cette hétérogénéité, les reboiseurs des régions mentionnées auraient intérêt — du moins jusqu'à plus ample informé — à éviter l'emploi de cette source de graines.

Enfin, l'engouement suscité actuellement en France par le mélèze du Japon et qui s'explique par la croissance initiale rapide de cette espèce, mérite d'être nuancé. Le mélèze des Sudètes et surtout les hybrides danois accusent au cours des trois premières années après plantation une croissance en hauteur at moins aussi forte que celle du mélèze du Tapon, d'où une économie du même ordre sur les dégagements. De plus, en présence de gelées tardives, le mélèze du Japon se révèle beaucoup plus sensible, d'où une survie inférieure. On peut penser, enfin, que le mélèze des $\mathrm{Su}$ dètes maintiendra sa croissance plus longtemps que le mélèze du Japon.

\section{Conclusion}

Les plantations comparatives de provenances débouchent sur deux catégories de résultats:

- sur le plan scientifique, une meilleure connaissance de la variabilité infraspécifique,

- sur le plan pratique, des informations concernant les sources de graines pour le reboisement.

L'expérience de Coat-an-Hay permet, dès maintenant, d'avanver quelques hypothèses sur le regroupement des populations à l'intérieur de l'aire naturelle du mélèze. Il a été mis en évidence certaines liaisons entre différents caractères. En particulier, la croissance rapide semble liée à la tardiveté, que l'on se place au niveau des provenances ou des individus.

(1) Directeur de la Station d'Amélioration d'Humlebaeck. 
Sur le plan pratique, il est possible d'orienter le choix des sources de graines pour le reboisement des régions de hasse ou moyenne altitude soumises à un climat océanique.

Avant de se prononcer de façon définitive sur les conclusions qui viennent d'être exposées, il faudra attendre que l'expérience arrive à son terme (40 ans); mais comme il arrive sonvent, en matière d'expérimentation forestière, on est amené à présenter des résultats provisoires.

\section{BIBLIOGRAPHIE}

1. Baldwin (H.-I.). - The period of height growth in different provenances of european larch. $12^{\mathrm{e}}$ Congrès IUFRO - Oxford, 1956.

2. Bouvarel (P.) et Lemoine (M.). - Races de mélèze et grosseur des graines.

Rezue Forestière Française, mai 1958, p. 348-350.

3. Duchaufour (Ph.). - Etudes sur l'écologie et la sylviculture du Mélèze (Larix europaea D.C.).

II. - Pédologie et facteurs biotiques.

Annales de l'Ecole Nationale des Eaux et Forêts et de la Station de Recherches et Expériences. T. XIII, fasc. 1, 1952, p. 135-203.

4. Fourchy (P.). - Etudes sur l'écologie et la sylviculture du mélèze (Larix europaea D.C.).

I. Ecologie du mélèze particulièrement dans les Alpes françaises. Annales de l'Ecole Nationale des Eaux et Forêts et de la Station de Recherches et Expériences. T. XIII, fasc. 1, 1952, p. 3-132.

5. Gathy (P.). - A propos du mélèze d'Europe en Belgique. Bulletin de la Société Royale Forestière de Belgique, août-septembre 1955.

6. Gathy (P.). - Contribution à l'étude des races de mélèze d'Europe. Travaux Série B, $\mathrm{n}^{\circ} 22$, Groenendaal, 1959.

7. Genys (B.-G.). - Geographic variation in European Larch. New Hampshire Forestry and Recreation Commission. Concord N.H., 1960.

8. Kiellander (C.-L.). - Report on the development of the international provenance test of larch in Sweden. Congrès IUFRO, Oxford, 1956.

9. Мс Сомв (A.-L.). - The european larch: its races, site requirements and characteristics.

Forest Science, I, 4 p., 298-318, 1955.

10. PARdé (J.). - Plaidoyer pour le mélèze. Revue Forestière Française, août-septembre 1957, p. 634-651.

11. Schober (R.). - Ergebnisse von Lärchen. Art- und Provenienzversuchen. Silvae Genetica 7, septembre-octobre 1958, p. 137-168.

12. Tschermak (L.). - Aus der Heimat der europäischen Lärche. Forstarchiv, 1932, p. 14-49.

13. Tschermak (L.). - Die natürliche Verbreitung der Lärche in der Ostalpen.

Mitt. a. d. F. Vw Oesterreichs, H. 43, 1935.

14. Wood (R.-F.) et Lines (R.). - Report on Forest Research for the year ended March 1958, p. 53-54.

15. Compte rendu de la réunion de travail des participants à l'expérience internationale de mélèzes à Hann-Münden, 13 au 18 octobre 1959. 


\section{RÉSUME}

L'étude a pour but de présenter les premiers résultats obtenus dans une plantation comparative de provenances de mélèze, installée en Bretagne, dans le cadre d'une expérimentation internationale organisée par le Professeur Schober.

Six ans après semis, il est possible de déceler des différences importantes concernant les caractères de survie, hauteur et précocité.

Il a été possible d'établir des liaisons entre ces caractères et de présenter un groupement des provenances en fonction de leur comportement et de leur situation géographique.

Il apparait qu'en zone océanique, les reboiseurs auront tout intérêt à s'approvisionner en graines dans les massifs des Sudètes, en Pologne, ou mieux d'utiliser l'hybride Larix eurolepis. 


\section{SUMMARY}

The study intends to present the first results obtained in Bretagne from a comparative study of plantations of larch of different origine. The study took place within the framework of an international experiment organized by Professor Schober.

Six years after the sowing, important differences may be noted between the characteristics of vigour, height and precocity.

It was possible to establish connections between some features and to arrange groups of different provenance, according to the behaviour and geographic position.

It seems that in the oceanic zone the creators of new plantations will use with profit seeds from the Sudeten (Poland), or better still the hybrid Larix eurolepis. 


\section{ZUSAMMENFASSUNG}

In diesem Beitrag werden die ersten Ergebnisse der Versuche mitgeteilt, die für Lärchenprovenienzen auf einer Versuchsfläche in der Bretagne durchgeführt worden sind. Diese auf zwischenstaatlicher Ebene veranstalten Versuche stehen unter der Leitung des Herrn Professor ScHober.

Sechs Jahre nach der Saat ist es möglich hinsichtlich der Lebenskraft, der Höhe und der frühen Reife beträchtliche Unterschiede festzustellen.

Man hat Beziehungen zwischen Merkmalen aufweisen, und die Herkunft je nach der Entwicklung und der geographischen Lage gruppenweise anordnen können.

Es hat sich herausgestellt, dass die Samen aus den Sudeten (Polen) für die Aufforstung der ozeanischen Zone am wertvollsten sind, oder besser noch die Bastardpflanze Larix eurolepis. 